\title{
Intra-aortic extension of ductal tissue
}

\author{
A. Fraisse, MD, E. Le Bret, MD, T. Abdel Massih, MD, A. Batisse, MD, F. Laborde, MD, D. Sidi, MD, and \\ D. Bonnet, MD, Paris, France
}

I $\mathrm{n}$ neonates, an intra-aortic mass in the distal arch or descending aorta is likely to be an aortic thrombosis, a widely recognized complication of umbilical artery catheterization. ${ }^{1}$ The mass can cause obstruction of the lumen, presenting with symptoms of coarctation, ${ }^{2}$ or embolization. The diagnosis and the origin of the mass can be confirmed by echocardiography with a high sensitivity and specificity. ${ }^{3}$ We report the case of a neonate with an intra-aortic mass in whom surgical removal and histologic examination of the mass revealed ductus arteriosus tissue.

\section{Case Report}

A 1-month-old male infant was transferred to the department of pediatric cardiology for management of an intra-aortic mass. He was born at 39 weeks' gestation, by cesarean section, and was subsequently admitted to the neonatology unit for meconium inhalation with transient polypnea, hypoglycemia, macrosomia (4.05 $\mathrm{kg}$ ), and presumed infection. He received intravenous antibiotics through a peripheral vein and was discharged home at 10 days of age after an uncomplicated hospital course. An echocardiogram, scheduled to rule out hypertrophic cardiomyopathy in the setting of macrosomia, showed a normal intracardiac anatomy and structure but revealed an echogenic intra-aortic mass (Figure 1, $A$ ). The bright and smooth borders of the mass were similar to those of an aortic thrombus, but the general appearance was heterogeneous. The mass seemed to be loosely attached to the aortic wall through a pedicle, beginning at a site corresponding to the aortic isthmus and extending toward the descending aorta. Physical examination at admission was normal. Blood pressure in each of the arms was $70 / 40 \mathrm{~mm} \mathrm{Hg}$, without decreased blood pressure in the legs. Magnetic resonance imaging confirmed the presence of a $2-\mathrm{cm}$

From the Service de Cardiologie Pédiatrique, Hôpital Necker-Enfants Malades, Paris, France.

Received for publication Oct 10, 2001; accepted for publication Nov 20, 2001.

Address for reprints: A. Fraisse, MD, Service de Cardiologie Pédiatrique, Hôpital Necker Enfants Malades, 149 rue de Sèvres, 75743 Paris Cedex 15, France (E-mail: afraisse@ mail.ap-hm.fr).

J Thorac Cardiovasc Surg 2002;123:568-9

Copyright $\odot 2002$ by The American Association for Thoracic Surgery

$0022-5223 / 2002 \$ 35.00+0 \quad \mathbf{1 2 / 5 4 / 1 2 1 7 5 9}$

doi: $10.1067 / \mathrm{mtc} .2002 .121759$ long and $0.5-\mathrm{cm}$ wide globular heterogeneous mass localized on the anterior aortic wall and attached to the isthmus (Figure 1, B). Although the patient was free of symptoms, surgical removal of the mass was recommended because of the risk of aortic obstruction or embolization.

Surgery was performed through a left posterolateral thoracotomy. The intra-aortic mass, connected to the occluded aortic end of the ductus arteriosus with a pedicle, was resected through a longitudinal opening in the aorta (Figure 2, $A$ and $B$ ). Aortic crossclamping time was 20 minutes. The postoperative course was uneventful and the patient was discharged home on the fifth postoperative day. Postoperative echocardiography confirmed the patency of the aorta. Histologic examination revealed acellular elastic fibrous tissue with numerous calcifications, consistent with involuted ductus arteriosus tissue (Figure 2, $C$ and $D$ ). No thrombus or fibrinous clot was observed. The patient is doing well 3 months after the operation.

\section{Comment}

Echocardiography can usually identify the size, attachments, consistency, and texture of intra-aortic masses. Its sensitivity and specificity for thrombus detection have been estimated to be as high as $95 \%$ and $86 \%$, respectively. ${ }^{3}$ Magnetic resonance imaging is also extremely valuable in the evaluation of intravascular structures and masses. ${ }^{2}$ In our patient, despite the heterogeneous appearance of the mass, the echocardiographic and magnetic resonance imaging appearance favored an aortic thrombus because of its bright and smooth borders. Moreover, the descending aorta, where the mass was located, is an area likely to harbor thrombi, even in the newborn without previous umbilical artery catheterization. ${ }^{4}$

Surgical removal of the mass was chosen in our patient becauseof (1) the important size of the mass, although there were no signs of aortic obstruction, (2) the risk of embolization favored by the heterogeneous appearance of the mass and the looseness of its attachments to the aortic wall, and (3) its unknown origin (clot or tumor). However, one can speculate that this asymptomatic tumor might have involuted such that surgical resection would not have been necessary.

Intra-aortic extension of ductus arteriosus tissue, as in our case, is very atypical and to our knowledge has not previously been reported. After birth, the ductus arteriosus usually closes within the first 15 hours after delivery, although it may persist, especially in cases of prematurity or hypoxemia, in a wide variety of shapes and sizes. Ductus arteriosus tissue may also extend toward the aorta 


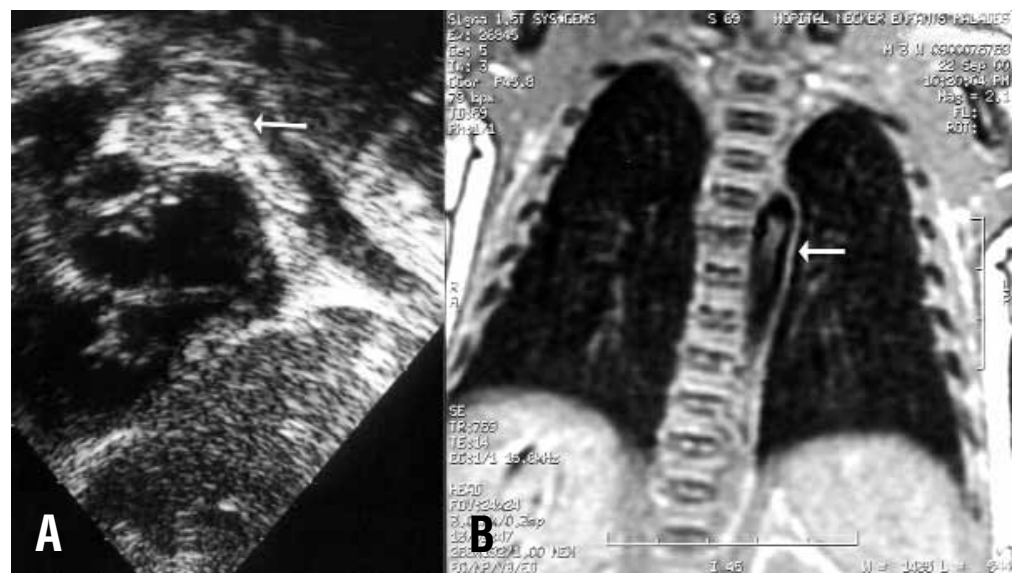

Figure 1. A, Echography of the descending aorta with the "tumor" attached to the isthmus. B, Magnetic resonance image of the descending aorta with the "tumor" floating in the aortic lumen.
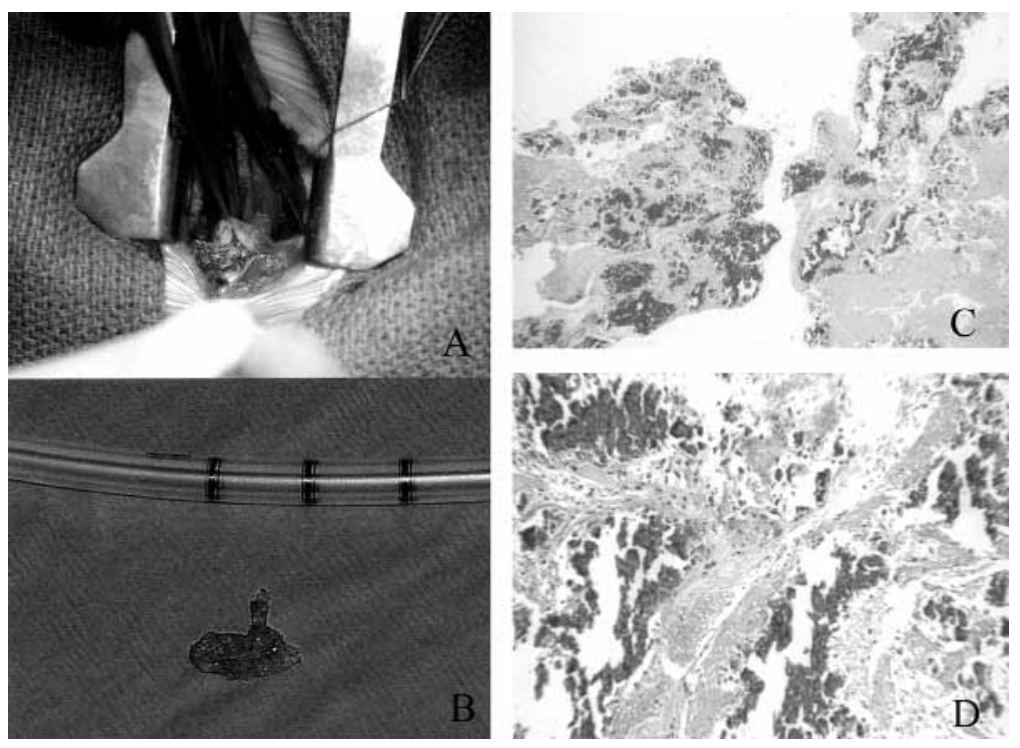

Figure 2. A, Operating view of the tumor after opening of the aorta. B, The "tumor" after removal. C and D, Histologic sections of the tumor. Note that the tumor was composed of ground substance with numerous calcifications, consistent with involuted ductus arteriosus tissue.

and contribute to coarctation with its closure, or toward the pulmonary arteries, inducing proximal stenoses. ${ }^{5}$ Usually, the combined effect of the increased oxygen and decreased circulating prostaglandins results in a contraction of the smooth muscles within the ductal walls, followed by enfolding of the endothelium and disruption of the intima leading to fibrosis, scarring, and a permanent seal of the ductus. ${ }^{5}$

In conclusion, we report the case of a neonate with unusual intra-aortic extension of ductus arteriosus tissue causing a heterogeneous intra-aortic mass. Surgical removal was recommended because of the potential risk of aortic obstruction or embolization and also because neither echocardiography nor magnetic resonance imaging could determine the origin of this intra-aortic mass.

\section{References}

1. Rodriguez M, Sosenko I. Catheter-induced aortic thrombus masquerading as coarctation of the aorta. Clin Pediatr. 1989;28:581-4.

2. Starc TJ, Abramson SJ, Bierman FZ, Regan JA, Sitarz AL, Rose EA, et al. Acquired coarctation of the aorta. Pediatr Cardiol. 1992;13:336.

3. Snider AR, Serwer GA, Ritter SB. Abnormalities within the cardiac chambers, proximal vessels, and thorax. In: Echocardiography in pediatric heart disease. St Louis: Mosby-Year Book; 1997. p. 497511.

4. Hamilton RM, Penkoske PA, Byrne P, Duncan NF. Spontaneous aortic thrombosis in a neonate presenting as coarctation. Ann Thorac Surg. 1988;45:564-5.

5. Mullins CE, Pagotto L. Patent ductus arteriosus. In: Garson A Jr, Bricker JT, Fisher DJ, Neish SR, editors: The science and practice of pediatric cardiology. Baltimore: Williams \& Wilkins; 1997;1181-97. 